\title{
Análisis de redes sociales y bibliométrico de las tesis españolas sobre drogodependencias en la base de datos TESEO
}

\section{Bibliometric and academic network analysis of Spanish theses on drug dependence in the TESEO database}

\author{
Lourdes Castelló i Cogollos*, Francisco Jesús Bueno Cañigral**, \\ Juan Carlos Valderrama Zurián****.
}

* Departament de Sociologia i Antropologia Social. Universitat de València; UISYS, Unidad Mixta de Investigación, CSIC, Universitat de València, Valencia, España. ** Plan Municipal de Drogodependencias; UPCCA Valencia. Concejalía de Sanidad, Salud y Deportes (Ayuntamiento de Valencia), Valencia, España. *** Instituto de Documentación y Tecnologías de la Información. INDOTEI. Universidad Católica de Valencia San Vicente Mártir, Valencia, España.

\section{Resumen}

El presente trabajo tiene por objeto desvelar la estructura de posiciones del campo de las adicciones a partir del análisis de las tesis doctorales defendidas en España. La fuente consultada para la selección de las tesis fue TESEO. La búsqueda se limitó al período 1976-2017 y se utilizaron los descriptores abuso de drogas, alcoholismo, efecto de las drogas y tratamiento de la drogadicción. Se obtuvieron 728 tesis, siendo el quinquenio más productivo el 2013-2017 con 208 (28,6\%) tesis doctorales. La evolución de las publicaciones es ascendente, excepto en el quinquenio 2003-2007 en el que la producción baja a un 5,2\% del total. La universidad que aporta más tesis es la Universitat de València. El académico que más tesis ha dirigido es Alfonso Velasco Martín y Emilio Ambrosio Flores es el investigador que más ha participado en los tribunales. El análisis de los actores participantes en las tesis doctorales muestra un incremento de presencia de las mujeres como autoras, directoras de tesis y miembros de tribunales. En relación a la temática, los temas más abordados han sido los relacionados con la psicología, la farmacología y las ciencias médicas. $\mathrm{El}$ análisis de las tesis doctorales sobre drogodependencias ha dado una visión de su estructura y de las personas más influyentes de la misma. El aumento de la preocupación social en esta área y la creación de grupos y unidades de investigación específicos ha ocasionado un incremento de la producción científica.

Palabras clave: Tesis académicas; Trastornos relacionados con sustancias; Bibliometría; Análisis de redes sociales; Análisis de género.

\begin{abstract}
The present study aims to reveal the structure of positions in the field of addiction through the analysis of doctoral theses read in Spain. The source consulted for the selection of the theses was TESEO. Searching for the keywords drug abuse, alcoholism, drug effects and drug addiction treatment during the period 1976-2017 produced 728 theses. The most productive period is 2013-2017, with 208 (28.6\%) doctoral theses. The overall rate of publication is increasing, but in the period 2003-2007 output decreased significantly to $5.2 \%$ of the total. The university contributing the most theses is the University of Valencia. The academic who has supervised the most theses is Alfonso Velasco Martín, and Emilio Ambrosio Flores is the researcher who has participated most on examining committees. The analysis of the participants in the doctoral thesis process shows a gradual increase of women in authorship, in thesis supervision and as examiners. In terms of subject, the most frequently treated aspects are those related to psychology, pharmacology and medical sciences.

The analysis of doctoral theses on substance abuse provides an overview of the structure and the most influential participants in this area. Increasing public concern and the creation of specific groups and research units are reflected in the growth of scientific output. Keywords: Academic Dissertations; Gender identity; Substance-Related Disorders; Bibliometrics; Social network analysis; Gender analysis.
\end{abstract}




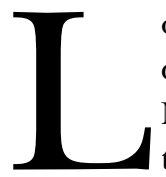

os informes anuales del Observatorio Europeo de las Drogas y las Toxicomanías (European Monitoring Centre for Drugs and Drug Addiction, 2018) muestran que España se encuentra entre los países de la Unión Europea con mayor consumo de cocaína y cannabis. Ello ha generado un aumento de la investigación sobre esta problemática, convirtiéndose en un área prioritaria para los organismos e instituciones (González-Alcaide et al., 2008; Melero-Fuentes, 2016; Vidal-Infer, 2010). Este incremento de la investigación se ve reflejado en la producción científica que ha ido creciendo paralelamente a la consolidación de este campo de investigación (Osca-Lluch, 2016) y ha impulsado la generación de estudios bibliométricos que caracterizan esta área por temática, sustancias, sexo de los autores o áreas geográficas (Bramness, Henriksen, Person y Mann, 2014; González, Fernandes, Pinto y Agulló, 2015; González-Alcaide et al., 2007; Khalili et al., 2018; Osca-Lluch, 2012, 2016). Sin embargo, estos estudios se han centrado sólo en el análisis de los artículos de revistas cuando se ha demostrado que la producción de tesis doctorales es proporcional a los artículos científicos indexados en bases de datos internacionales, tanto en su crecimiento a lo largo del tiempo, como en su distribución entre las universidades (Sánchez, Blázquez, Montesi y Botezan, 2017).

La defensa de la tesis doctoral es la actividad culminante del programa de doctorado (MacLennan, Piña y Gibbons, 2018), siendo el grado de doctor la mayor titulación que se puede lograr en el ámbito universitario (Curiel-Marín y Fernández-Cano, 2015; Curiel-Marín, Passoni, Olmedo-Moreno y Fernández-Cano, 2018). Mediante el desarrollo y la elaboración de la tesis doctoral, los doctorandos se dotan de los conocimientos metodológicos y habilidades analíticas necesarias para producir y difundir los resultados de la investigación (Davis, Wladkowski y Mirick, 2017), para desarrollar una carrera en el mundo académico (Piña, MacLennan, Moran y Hafford, 2016) y para ser trabajadores altamente cualificados e investigadores competentes en los diferentes campos académicos (Caparrós-Ruíz, 2018).

El número de tesis doctorales dirigidas es un indicador para llevar a cabo un doctorado con éxito (Buela, 2005) e influye en el prestigio de los profesores y en su carrera académica. De hecho, el programa Academia para la acreditación del profesorado de la Agencia Nacional de Evaluación de la Calidad y Acreditación (ANECA), incluye como criterios de evaluación, tanto de la actividad investigadora como de la actividad docente, el haber sido director de tesis doctorales (ANECA, 2017). Cabe resaltar que las tesis doctorales constituyen uno de los indicadores del informe "Las Universidades españolas en cifras" realizado por la Conferencia de Rectores de Universidades Españolas (CRUE, 2017), y también los títulos de doctor concedidos se utilizan como criterios de evaluación en diversos rankings de universidades que se elaboran, como el RUR World University Ranking (Round University Ranking, 2018), THE World University Ranking (Times Higher Educación, 2018) o el U-Multirank. Universities Compared. Your way (U-Multirank, 2018).

Por tanto, el análisis de las tesis doctorales es un buen indicador para dilucidar las líneas y tendencias de investigación en un campo de la ciencia y permite una aproximación a la estructura social de esa disciplina, pues en el proceso de elaboración y evaluación participan profesionales con roles establecidos: autores, directores, revisores y miembros del tribunal (Arguimbau-Vivó y Fuentes-Pujol, 2009; Delgado, Torres-Salinas, Jiménez-Contreras y Ruiz-Pérez, 2006; Repiso, Torres y Delgado, 2011a)

El presente trabajo tiene por objeto desvelar la estructura de posiciones de los académicos del campo de las adicciones a partir del análisis bibliométrico y de redes sociales de las tesis doctorales defendidas en España y que están incluidas en la base de datos TESEO (https://www.educacion.gob.es/teseo/irGestionarConsulta.do), aportando una información complementaria a los resultados obtenidos en estudios bibliométricos de artículos científicos del área de las adicciones. Para ello, se toma como referencia las tesis defendidas, sus autores, los directores y los componentes de los tribunales de evaluación, el sexo de los actores implicados y la temática de las tesis.

Un avance de este estudio (Castelló-Cogollos, Bueno y Aleixandre, 2016) se publicó como nota breve en la sección de "Temas de Actualidad" del centro de documentación CendocBogani, realizándose en el presente artículo un análisis más amplio y en profundidad de los datos obtenidos para dicha nota.

\section{Metodología}

La fuente consultada para la selección de las tesis fue TESEO, base de datos del Ministerio de Educación, Cultura y Deporte que contiene información de las tesis defendidas en España desde 1976, clasificados según los códigos del tesauro de la UNESCO (https://es.unesco.org/).

La ecuación de búsqueda para la recuperación de las tesis doctorales sobre Adicciones incluyo los descriptores: "abuso de drogas", "alcoholismo", "efecto de las drogas" y "tratamiento de la drogadicción". Se realizaron búsquedas independientes con cada uno de los descriptores, y por cada uno de los cursos académicos desde 1976 a 2017. El último curso académico incluido en la ecuación de búsqueda fue 2017/2018, seleccionándose, en este caso, las tesis doctorales que se habían defendido en el año 2017. En total se obtuvieron 832 registros que fueron descargados e incluidos en un una base de datos relacional en $\mathrm{Mi}$ crosoft Access creada "ad hoc" para el presente estudio y que incluía título de la tesis, descriptores, autor, institución dónde se defendió la tesis, director o directores de la tesis y 
miembros del tribunal, especificando el papel que desempeñan en el tribunal.

Al realizar búsquedas individuales para cada uno de los descriptores se detectaron duplicados, ya que los registros tenían dos o más descriptores utilizados para la búsqueda. La muestra definitiva, una vez eliminados los duplicados, estaba constituida por 728 tesis. Posteriormente se procedió a la homogeneización de las afiliaciones institucionales, las firmas de los autores, directores y miembros del tribunal y los descriptores. Se asignó el sexo a cada uno de los actores participantes en la realización y defensa de la tesis.

El análisis bibliométrico se ha dividido en cinco grandes bloques: a) análisis de autorías, en el que se exponen cálculos sobre las autorías de las tesis; b) análisis de los directores, como director único o codirector, calculándose el índice de codirección (total de tesis dirigidas entre el total de tesis); c) análisis de tribunales, en el que se presentan los datos referentes a los miembros de tribunal que participan en las tesis; d) comparativa según el sexo, en la que se cotejan los resultados por sexo de los tres actores que participan en las tesis (autores, directores y miembros del tribunal), y e) análisis temático, en el que se analiza la frecuencia de los descriptores asignados a las tesis y sus relaciones.

Para la creación y visualización gráfica de las redes de descriptores, tribunales y del binomio director-miembro del tribunal se utilizó el programa Pajek (http://mrvar.fdv. uni-lj.si/pajek/). El tamaño de las esferas es proporcional al número de ocurrencias. El grosor de las líneas que conectan dos esferas son proporcionales al número de veces que los dos términos aparecen simultáneamente en el total de tesis. Para la representación gráfica de los descriptores se consideró la co-ocurrencia de tres o más descriptores en la misma tesis doctoral y para las redes de miembros de los tribunales se consideró la co-ocurrencia en tres o más tesis doctorales.

La red de invitación Director-Tribunal proporciona una visión más próxima de la existencia de relaciones intensas entre los académicos, ya que en ella se representan las veces que un director invita, recomienda o requiere la presencia de un académico para participar como tribunal de la tesis doctoral que ha dirigido. Para ello, se ha considerado establecer como relación la invitación por parte del director de la tesis a distintos académicos para formar parte del tribunal. Se ha realizado así, porque se ha tenido en cuenta el reglamento sobre el depósito, evaluación y defensa de la tesis de diversas universidades, entre ellas la Universidad Complutense de Madrid y la Universitat de València, en los que se expone que se debe entregar, junto con el depósito de la tesis, una lista de seis expertos para su evaluación. Anteriormente eran diez expertos, pero se ha ido modificando desde la publicación del Real Decreto 99/2011, de 28 de enero, por el que se regulan las enseñanzas oficiales de doctorado (Real Decreto 99/2011, 2011). Esto indica que la selección de las personas para formar parte del tribunal de la tesis la realiza su director o directores y, por tanto, revela una relación entre los directores y los miembros del tribunal. En la elaboración de esta red se consideró la co-ocurrencia en tres o más tesis doctorales

\section{Resultados}

\section{Datos globales}

La primera tesis doctoral sobre drogodependencias incluida en TESEO es "Aspectos psicológicos y evaluativos de la personalidad alcohólica” de Flora Paniagua Cárdenas, defendida en 1978 en la Universidad Complutense de Madrid. El quinquenio más productivo es el 2013-2017 con 208 $(28,6 \%)$ tesis doctorales (figura 1). Aunque la evolución general de la producción de tesis doctorales es ascendente, en el quinquenio 2003-2007 baja significativamente, situándose en un 5,2\% del total, 11,4 puntos porcentuales menos que en el quinquenio anterior $(16,6 \%)$. Se observa, que el $11,7 \%$ de los autores de las tesis participan posteriormente como directores o miembros de tribunal y que 22 autores han participado como autor, director y miembro de tribunal.

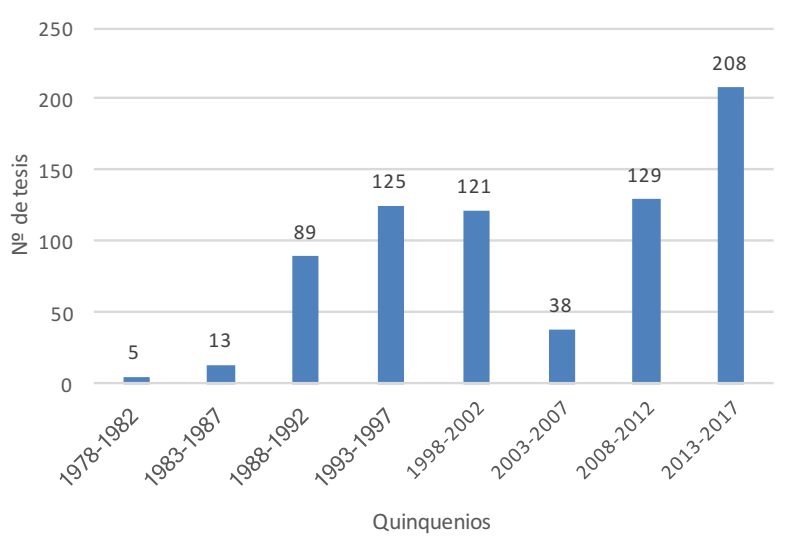

Figura 1. Evolución por quinquenios de la producción de tesis doctorales españolas sobre drogodependencias.

El mayor número de tesis doctorales han sido defendidas en Cataluña (16,8\%), seguida de Madrid y Comunidad Valenciana, con un $15,7 \%$ y $15,4 \%$, respectivamente. En Andalucía se han defendido el $13,9 \%$ de las tesis doctorales. Las tesis doctorales se han defendido en 51 universidades. La Universitat de València $(n=69)$ es la que más tesis aporta al estudio, seguida de la Universitat Autònoma de Barcelona $(n=57)$ y la Universidad de Valladolid con 51 tesis; también se encuentra en los primeros puestos la Universidad Complutense de Madrid con 46 tesis. En la tabla 1 se observan las universidades en las que se han defendido 10 o más tesis. 
Tabla 1. Distribución de las tesis doctorales españolas sobre drogodependencias por universidades de defensa ( $\geq$ de 10 tesis ordenadas por número de tesis defendidas).

\begin{tabular}{|c|c|c|}
\hline Universidad de defensa & № de tesis & $\%$ \\
\hline Universitat de València (Estudi General) & 69 & $9,5 \%$ \\
\hline Universidad Autónoma de Barcelona & 57 & $7,8 \%$ \\
\hline Universidad de Valladolid & 51 & $7,0 \%$ \\
\hline Universidad Complutense de Madrid & 46 & $6,3 \%$ \\
\hline Universidad de Santiago de Compostela & 38 & $5,2 \%$ \\
\hline Universidad de Barcelona & 36 & $4,9 \%$ \\
\hline Universidad de Granada & 34 & $4,7 \%$ \\
\hline Universidad de Murcia & 29 & $4,0 \%$ \\
\hline $\begin{array}{l}\text { Universidad del País Vasco/ } \\
\text { Euskal Herriko Unibertsitatea }\end{array}$ & 29 & $4,0 \%$ \\
\hline Universidad Autónoma de Madrid & 25 & $3,4 \%$ \\
\hline Universidad de La Laguna & 24 & $3,3 \%$ \\
\hline Universidad de Salamanca & 23 & $3,2 \%$ \\
\hline Universidad de Sevilla & 23 & $3,2 \%$ \\
\hline Universidad de Málaga & 19 & $2,6 \%$ \\
\hline Universidad Miguel Hernández de Elche & 18 & $2,5 \%$ \\
\hline Universidad de Zaragoza & 17 & $2,3 \%$ \\
\hline Universidad Pompeu Fabra & 17 & $2,3 \%$ \\
\hline Universidad Nacional de Educación a Distancia & 15 & $2,1 \%$ \\
\hline Universidad de Cádiz & 13 & $1,8 \%$ \\
\hline Universidad de Oviedo & 12 & $1,6 \%$ \\
\hline Universidad de Navarra & 10 & $1,4 \%$ \\
\hline Universidad Jaume I de Castellón & 10 & $1,4 \%$ \\
\hline
\end{tabular}

\section{Análisis de los directores de las tesis}

$\mathrm{El}$ análisis de los directores de las tesis muestra que hay 664 directores o codirectores diferentes. Este análisis se ha realizado con las tesis que poseían esta información en el registro de la base de datos TESEO $(n=663 ; 91,1 \%$ del total de tesis). El índice de codirección es de 1,54 directores. El académico que más tesis ha dirigido es Alfonso Velasco Martín ( $n=26$ ) catedrático de farmacología la Universidad de Valladolid; le siguen, con una diferencia significativa, Emilio González Reimers, catedrático del área de medicina interna de la Universidad de la Laguna, con 11 tesis dirigidas. Del total de directores, el 75,9\% han dirigido solamente una tesis, el $20 \%$ entre dos y cuatro tesis y el $4,1 \%$ cinco o más tesis. En la tabla 2 se presentan los académicos que han dirigido cinco o más tesis doctorales.

La red de directores-tribunales muestra que participan 106 académicos y se crean 22 grupos, siendo el grupo más numeroso el formado por 28 componentes (figura 2). En ella se observa como no existe reciprocidad a la hora de recomendar los directores a otro académico a formar parte de los tribunales, pero si se aprecia que existe una relación significativa alrededor de Alfonso Velasco Martín que reco-
Tabla 2. Distribución de los directores de las tesis doctorales españolas sobre drogodependencias ( $\geq$ de 5 tesis dirigidas ordenadas por número de tesis dirigidas y alfabéticamente).

\begin{tabular}{|c|c|c|c|}
\hline Nombre & Director/a & Codirector/a & $\begin{array}{c}\text { № de tesis } \\
\text { dirigidas }\end{array}$ \\
\hline Velasco Martín, Alfonso & 25 & 1 & 26 \\
\hline Gonzalez Reimers, Emilio & 10 & 1 & 11 \\
\hline Bermejo Barrera, Ana María & 8 & 1 & 9 \\
\hline Luna Maldonado, Aurelio & 8 & 1 & 9 \\
\hline Miñarro López, José & 6 & 3 & 9 \\
\hline Álvarez González, Javier & 8 & & 8 \\
\hline Farré Albaladejo, Magí & 6 & 2 & 8 \\
\hline Romero Gómez, Francisco Javier & 7 & 1 & 8 \\
\hline $\begin{array}{l}\text { Santolaria Fernández, } \\
\text { Francisco Javier }\end{array}$ & 7 & 1 & 8 \\
\hline de la Torre Fornell, Rafael & 3 & 3 & 7 \\
\hline $\begin{array}{l}\text { García Del Castillo Rodríguez, } \\
\text { José Antonio }\end{array}$ & 6 & 1 & 7 \\
\hline Ambrosio Flores, Emilio & 5 & 1 & 6 \\
\hline Cadaveira Mahía, Fernando & 4 & 2 & 6 \\
\hline Fernández Gómez, Purificación & 3 & 3 & 6 \\
\hline Garcia Algar, Oscar & 4 & 2 & 6 \\
\hline Maldonado López, Rafael & 1 & 5 & 6 \\
\hline Pérez García, Miguel & 4 & 2 & 6 \\
\hline Rodríguez Arias, Marta & 3 & 3 & 6 \\
\hline Domingo Salvany, Antònia & 4 & 1 & 5 \\
\hline Espada Sánchez, José Pedro & 5 & & 5 \\
\hline Espí Martínez, Fernando & 5 & & 5 \\
\hline $\begin{array}{l}\text { González Aragón, } \\
\text { Carlos Manuel }\end{array}$ & 4 & 1 & 5 \\
\hline Graña Gómez, José Luis & 4 & 1 & 5 \\
\hline Luengo Martín, Maria Ángeles & 4 & 1 & 5 \\
\hline $\begin{array}{l}\text { Miquel Salgado-Araujo, } \\
\text { Marta Asunta }\end{array}$ & 3 & 2 & 5 \\
\hline Tabernero Duque, María Jesús & 2 & 3 & 5 \\
\hline Torrens Melich, Marta & 4 & 1 & 5 \\
\hline
\end{tabular}

mienda, en 11 ocasiones, a participar en los tribunales de las tesis en las que es director a Luis San Román del Barrio (Universidad de Salamanca) y a Fernando Fernández de la Gandara (Universidad Complutense de Madrid). También destaca la relación con Pedro Sánchez García (Universidad Autónoma de Madrid) o Antonio Dueñas Laita (Universidad de Valladolid). Asimismo, se pueden ver vértices (académicos) que hacen de nexo con otros académicos como, por ejemplo, Cecilio Álamo González (Universidad de Alcalá de Henares) o Francisco Javier Álvarez González (Universidad de Valladolid).

En la figura 3 que incluye cuatro grupos de seis o siete componentes, se observa reciprocidad en la relación formada por Emilio González Reimers y Francisco Javier Santolaria Fernández de la Universidad de La Laguna, que se 
invitan mutuamente a formar parte de los tribunales de las tesis en la que son directores. También existe reciprocidad en la relación entre José Antonio García del Castillo Rodríguez y José Pedro Espada Sánchez de la Universidad Miguel Hernández de Elche.

\section{Análisis de los tribunales de las tesis}

En los tribunales han intervenido 2.055 doctores distintos. Emilio Ambrosio Flores es el investigador que más ha participado en los tribunales (tabla 3) con un total de 20 asistencias (cinco como presidente, uno como secretario y

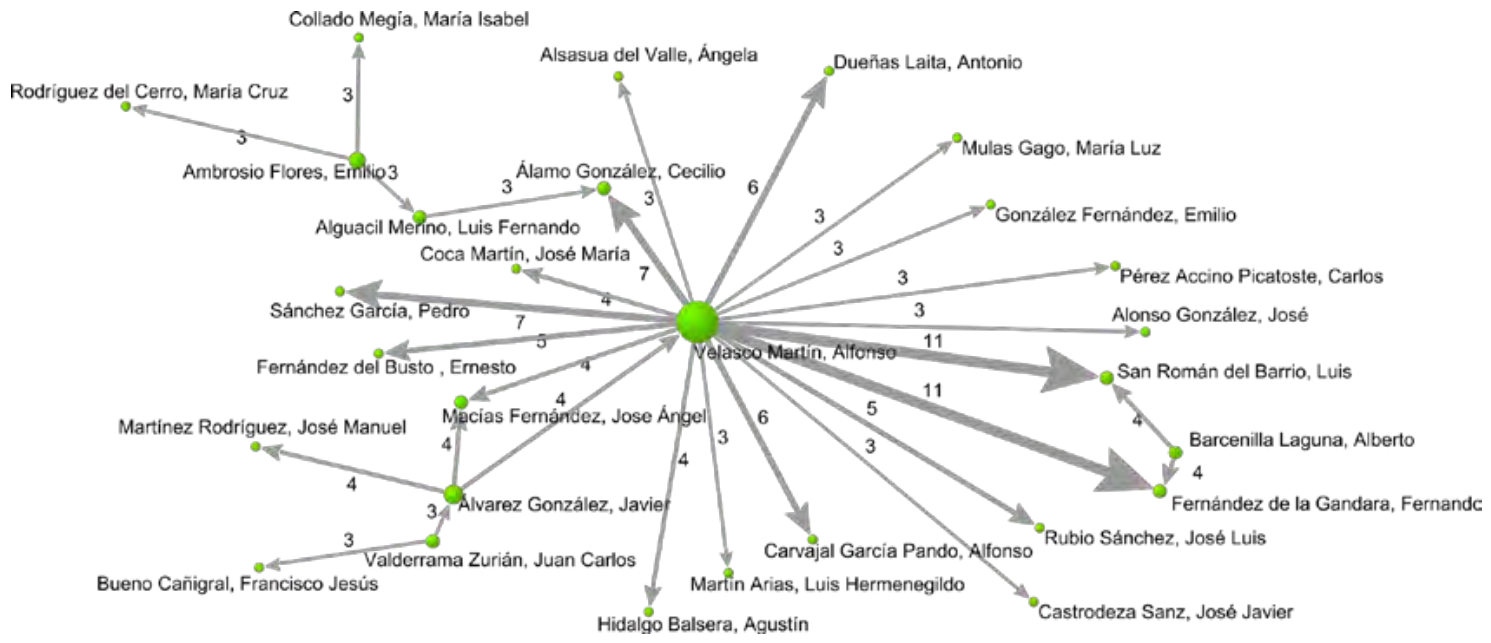

Figura 2. Red de invitación Director-Tribunal en las tesis doctorales españolas sobre drogodependencias (grupo de 28 componentes).

Nota. Los vértices representan a los académicos que ejercen como directores o tribunales de tesis. El grosor de los vértices determina las conexiones directas con otros vértices. El grosor de las flechas representa el total de veces que el director llama a participar en una tesis a un académico.
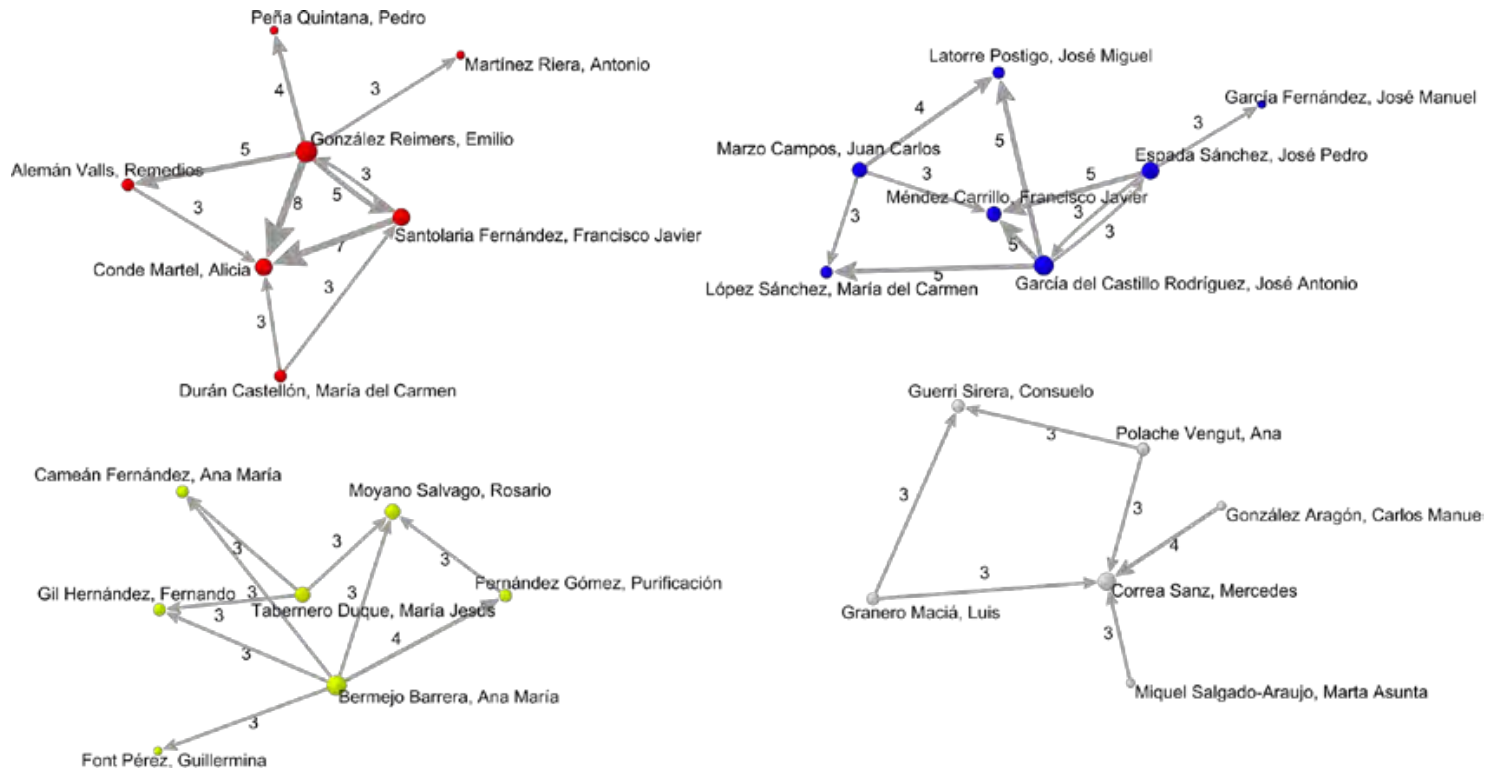

Figura 3. Red de invitación Director-Tribunal en las tesis doctorales españolas sobre drogodependencias (grupos de 7-6 componentes).

Nota. Los vértices representan a los académicos que ejercen como directores o tribunales de tesis. El grosor de los vértices determina las conexiones directas con otros vértices. El grosor de las flechas representa el total de veces que el director llama a participaren una tesis a un académico. 
Tabla 3. Distribución de los tribunales en las tesis doctorales españolas sobre drogodependencias ( $\geq$ de 10 asistencias a tribunales ordenados por número de asistencias a tribunales y alfabéticamente).

\begin{tabular}{|c|c|c|c|c|c|}
\hline Nombre & Presidente/a & Secretario/a & Vocal & $\begin{array}{c}\text { № de } \\
\text { asistencias a } \\
\text { tribunales }\end{array}$ & Centro de trabajo \\
\hline Ambrosio Flores, Emilio & 5 & 1 & 14 & 20 & Universidad Nacional de Educación a Distancia \\
\hline Sánchez Turet, Miguel & 8 & & 9 & 17 & Universitat de Barcelona \\
\hline Becoña Iglesias, Elisardo & 4 & & 12 & 16 & Universidad de Santiago de Compostela \\
\hline Casas Brugue, Miquel & 4 & 3 & 9 & 16 & Fundación Hospital Universitario Vall d`Hebron \\
\hline Conde Martel, Alicia & 1 & & 13 & 14 & Universidad de Las Palmas de Gran Canaria \\
\hline Alguacil Merino, Luis Fernando & 4 & & 9 & 13 & Universidad San Pau CEU \\
\hline San Román Del Barrio, Luis & 2 & & 11 & 13 & Universidad de Salamanca \\
\hline Torrens Melich, Marta & 4 & 3 & 6 & 13 & Institut Hospital del Mar d'Investigacions Mèdiques \\
\hline Álamo González, Cecilio & 4 & 1 & 7 & 12 & Universidad de Alcalá de Henares \\
\hline Collado Megía, Maria Isabel & 2 & 2 & 8 & 12 & Universidad Complutense de Madrid \\
\hline Fernández De La Gandara, Fernando & 5 & & 7 & 12 & Universidad Complutense de Madrid \\
\hline Simón Pérez, Vicente & 9 & 1 & 2 & 12 & Universidad de Valencia \\
\hline Valverde Granados, Olga & 4 & 3 & 5 & 12 & Universitat Pompeu Fabra \\
\hline Álvarez González, Javier & 2 & 3 & 6 & 11 & Universidad de Valladolid \\
\hline Dueñas Laita, Antonio & & 2 & 9 & 11 & Universidad de Valladolid \\
\hline Jané Carrenca, Francesc & 10 & & 1 & 11 & Universitat Autònoma de Barcelona \\
\hline Leal Cercós, Carmen & 3 & & 8 & 11 & Universitat de València \\
\hline Mendez Carrillo, Francisco Javier & 7 & & 4 & 11 & Universidad de Murcia \\
\hline Vega Fuente, Amando & 3 & & 8 & 11 & Universidad del País Vasco \\
\hline Echeburua Odriozola, Enrique & 7 & & 3 & 10 & Universidad del País Vasco \\
\hline
\end{tabular}

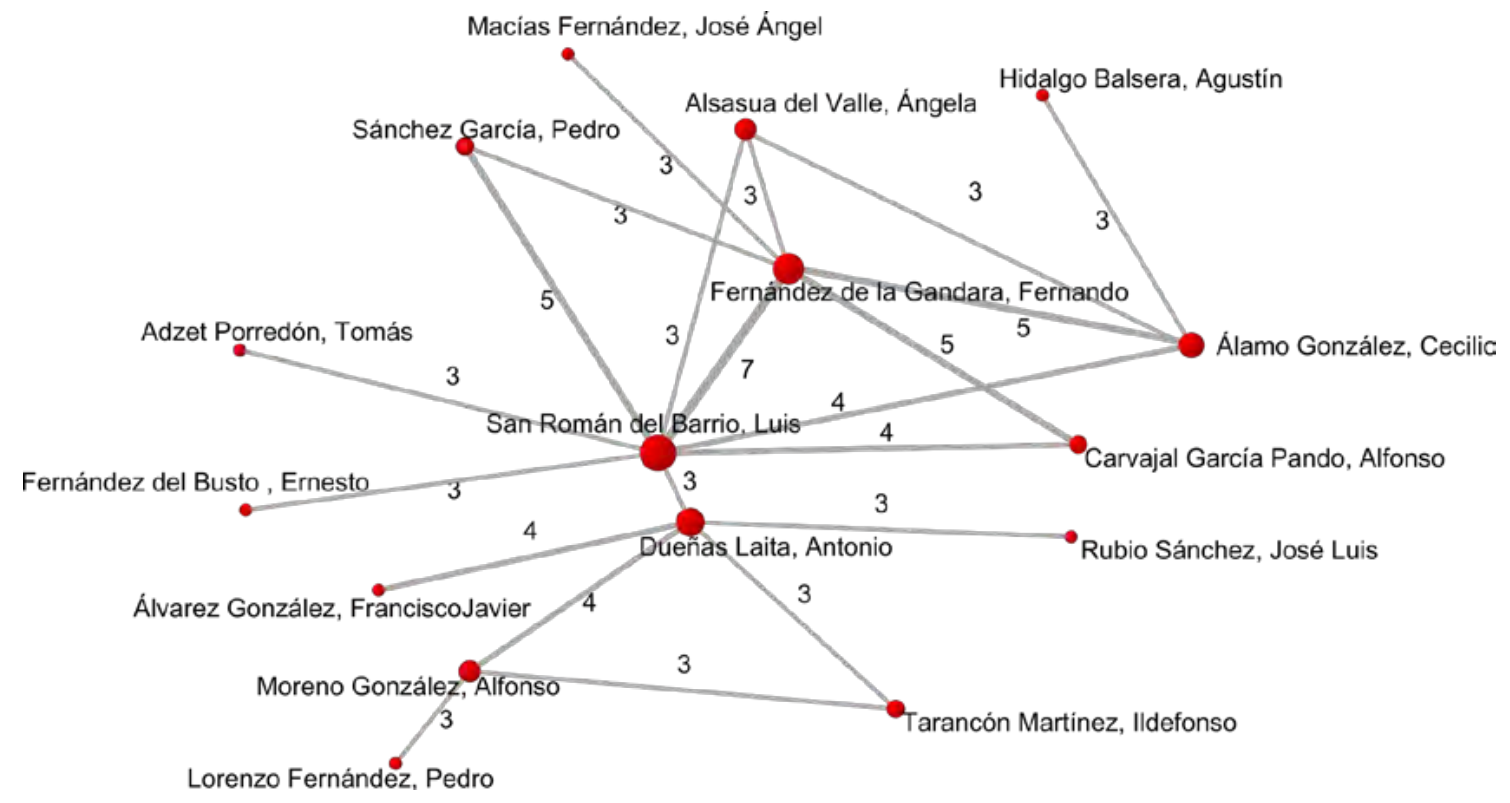

Figura 4. Red de tribunales en las tesis doctorales españolas sobre drogodependencias (grupo de 16 componentes).

Nota. Los vértices representan a los académicos que ejercen como directores o tribunales de tesis. El grosor de los vértices determina las conexiones directas con otros vértices. El grosor de las líneas representa el total de veces que co-ocurren dos tribunales en la misma tesis. 


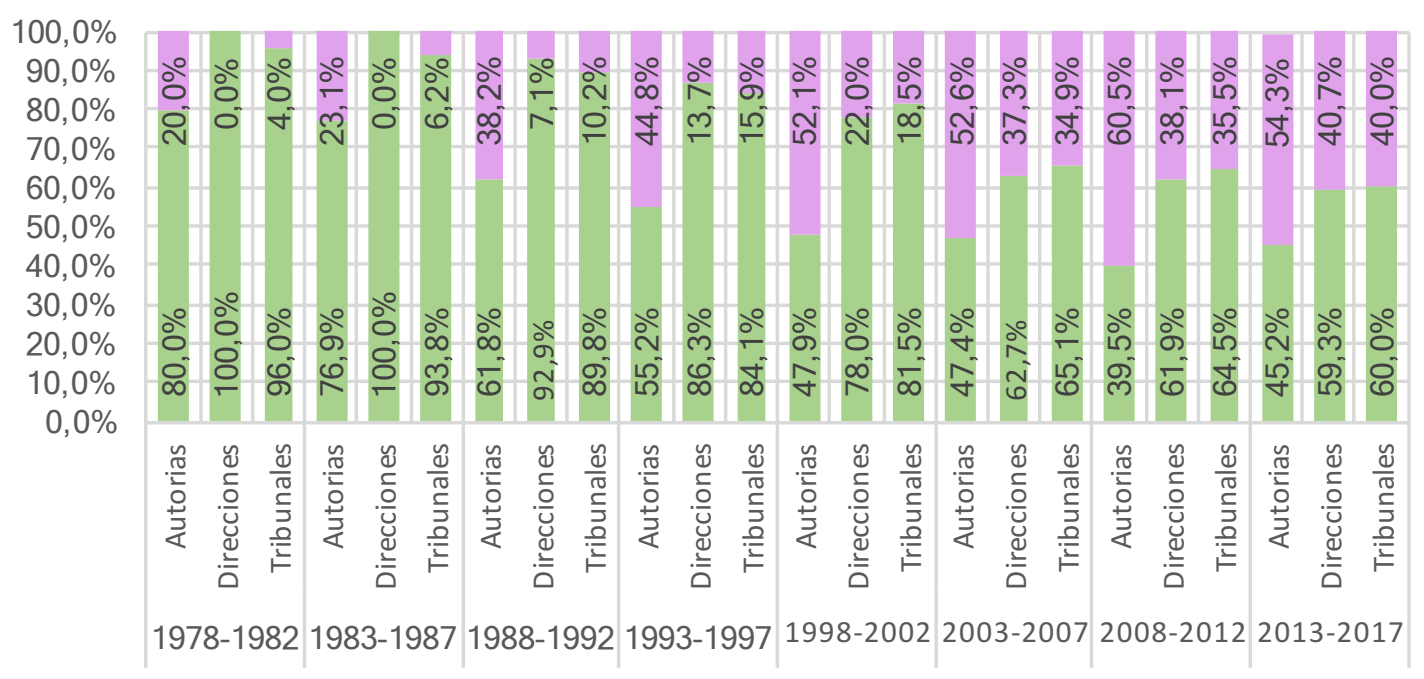

Hombres Mujeres

Figura 5. Comparativa según sexo de los actores participantes en las tesis doctorales sobre drogodependencias.

14 como vocal), a continuación se encuentra Miguel Sánchez Turet que ha asistido a 17 tribunales (ocho como presidente y nueve como vocal).

Si se considera que el cargo con el que se participa en un tribunal de tesis no tiene el mismo peso, se aprecia que es Francesc Jané $(n=10)$ el que más ha participado como presidente de tribunal, seguido de Vicente Simón Pérez $(\mathrm{n}=9)$ y Miguel Sánchez Turet $(\mathrm{n}=8)$. Asimismo, la mayoría de académicos que asisten a tribunales, lo hacen tan solo una vez $(74 \%)$ y solo $22(1,1 \%)$ de ellos asisten 10 o más veces (tabla 3 ).

El análisis de la red de los miembros de los tribunales muestra que se forma una red en la que participan 67 miembros de tribunal y se crean 21 grupos. El grupo más numeroso está formado por 16 componentes (figura 4) y muestra como Luis San Román del Barrio (Universidad de Salamanca) es el académico que más veces coincide con otros académicos en los tribunales de tesis. Además, éste co-ocurre más en los tribunales con Fernando Fernández de Gandara (Universidad Complutense de Madrid) y que ellos, a su vez, forman dos triángulos de co-presencias con Cecilio Álamo González (Universidad de Alcalá de Henares) y con Alfonso Carvajal García Pando (Universidad de Valladolid).

\section{Comparativa según el sexo de los actores participantes en las tesis}

El 49,5\% de las tesis han sido realizadas por hombres y el $50,5 \%$ por mujeres. En los cuatro primeros quinquenios el porcentaje de autoría de hombres es mayor que el de mujeres y a partir del quinquenio 1998-2002 la situación cambia y son las mujeres las que más tesis realizan. En este sentido, la diferencia más significativa se produce en el periodo 2008-2012, donde el porcentaje alcanzado por la producción femenina es del 60,5\% (figura 5).

Con respecto a los directores, el 68,2\% son hombres y el $31,8 \%$ mujeres. En la figura 5 se observa que hay un ascenso constante y paulatino de la participación de las mujeres como directoras de tesis doctorales, pasando de ninguna participación en el primer y segundo quinquenio al 40,7\% en el último quinquenio.

En el caso de los tribunales, los porcentajes de participación son del $74,4 \%$ de hombres y del $25,6 \%$ de mujeres, observándose una tendencia creciente en el porcentaje de mujeres que participan en los tribunales. El porcentaje de participación de las mujeres en los tribunales, durante los dos primeros lustros, es mayor que el porcentaje de tesis dirigidas por mujeres, con valores de $4 \%$ y el $6,2 \%$ en los tribunales y de cero como directora. Estas diferencias van desapareciendo progresivamente y en el quinquenio 19982002 el porcentaje de mujeres entre los directores supera ya al porcentaje de mujeres en los tribunales.

\section{Análisis temático de las tesis}

Los aspectos generales tratados en las tesis doctorales son la psicología, la farmacología y las ciencias médicas. Dentro del campo de la psicología, destaca psicofarmacología $(n=385)$ y psicología $(n=379)$, psicopatología $(n=31)$, psicología experimental $(n=23)$ y la psicología clínica y social con 21 tesis cada una. En las ciencias médicas destacan el descriptor ciencias médicas $(n=223)$, la psiquiatría $(n=52)$, la medicina interna $(n=37)$ y la salud pública $(n=35)$. Los descriptores específicos relacionados con las adicciones son abuso de drogas $(n=304)$, seguido de alcoholismo $(n=200)$, efecto de las drogas $(n=196)$ y tratamiento de la drogadicción $(n=126)$. En la tabla 4 se 




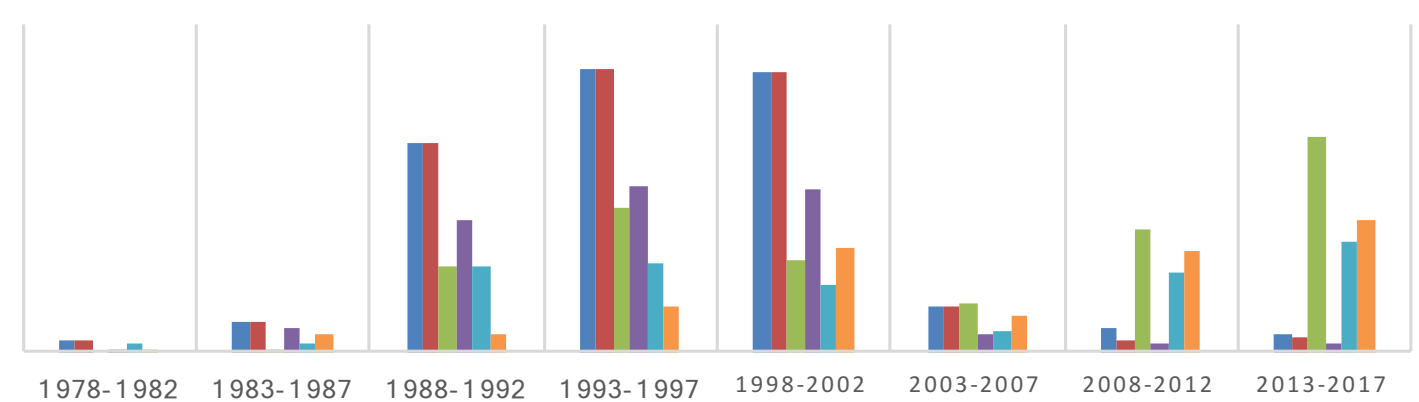

Figura 6. Evolución por quinquenio de los seis descriptores más empleados en las tesis doctorales sobre drogodependencias.

Tabla 4. Frecuencia de los descriptores en las tesis españolas sobre drogodependencias(> de 10 tesis doctorales)

\begin{tabular}{|c|c|c|}
\hline Descriptor & Frecuencia & $\%$ \\
\hline Psicofarmacología & 385 & $12,6 \%$ \\
\hline Psicología & 379 & $12,4 \%$ \\
\hline Abuso de drogas & 304 & $9,9 \%$ \\
\hline Ciencias médicas & 223 & $7,3 \%$ \\
\hline Alcoholismo & 200 & $6,5 \%$ \\
\hline Efecto de las drogas & 196 & $6,4 \%$ \\
\hline Tratamiento de la drogadicción & 126 & $4,1 \%$ \\
\hline Psiquiatría & 52 & $1,7 \%$ \\
\hline Farmacodinamia & 42 & $1,4 \%$ \\
\hline Farmacología & 40 & $1,3 \%$ \\
\hline Medicina interna & 37 & $1,2 \%$ \\
\hline Salud pública & 35 & $1,1 \%$ \\
\hline Mecanismos de acción de los fármacos & 33 & $1,1 \%$ \\
\hline Psicopatología & 31 & $1,0 \%$ \\
\hline Epidemiología & 28 & $0,9 \%$ \\
\hline Neurociencias & 27 & $0,9 \%$ \\
\hline Fármacos psicotrópicos & 27 & $0,9 \%$ \\
\hline Toxicología & 26 & $0,8 \%$ \\
\hline Psicología experimental & 23 & $0,8 \%$ \\
\hline Psicología clínica & 21 & $0,7 \%$ \\
\hline Psicología social & 21 & $0,7 \%$ \\
\hline Psicología del niño y del adolescente & 20 & $0,7 \%$ \\
\hline Medicina preventiva & 19 & $0,6 \%$ \\
\hline Ciencias clínicas & 18 & $0,6 \%$ \\
\hline Personalidad & 18 & $0,6 \%$ \\
\hline Ciencias de la vida & 17 & $0,6 \%$ \\
\hline Psicología de la anormalidad & 16 & $0,5 \%$ \\
\hline Psicología fisiològica & 16 & $0,5 \%$ \\
\hline Evaluación y diagnostico en psicologia & 13 & $0,4 \%$ \\
\hline Respuestas del comportamiento & 13 & $0,4 \%$ \\
\hline Patología & 13 & $0,4 \%$ \\
\hline Sociología & 13 & $0,4 \%$ \\
\hline Acción de los fármacos & 11 & $0,4 \%$ \\
\hline Neurología & 11 & $0,4 \%$ \\
\hline
\end{tabular}

presentan los descriptores que aparecen en más de 10 tesis doctorales.

En la figura 6 se presentan los seis descriptores más frecuentes en cada quinquenio. Se observa que en los primeros quinquenios las tesis doctorales se centran en aspectos relacionados con la psicología, teniendo su auge en los dos quinquenios centrales que abarcan desde el 1993 a 2002, y en los quinquenios posteriores sufre un descenso muy significativo hasta casi desaparecer. Los efectos de las drogas emerge con fuerza en los años que van de 1998 hasta 2002, y aunque posteriormente descienden ligeramente, en el último quinquenio se convierte junto al abuso de drogas en los aspectos más investigados y, por lo tanto, los que más preocupan, junto con el alcoholismo.

En las figuras 7 y 8 se presentan las relaciones de co-descriptores en una misma tesis, en los periodos 1978-1997 y 1998-2017. En la red del primer periodo (1978-1997) se observa que psicología y psicofarmacología aparecen más veces en las tesis con otros descriptores. Éstos a su vez, tienen co-presencias intensas con abuso de drogas, alcoholismo y ciencias médicas. Descriptores como efecto de las drogas, farmacodinamia o psiquiatría tienen relaciones de notable intensidad, mientras que descriptores relacionados con el comportamiento, el derecho o la sociología, entre otros, se encuentran en las periferias de la red.

En la red del segundo periodo (1998-2017) el abuso de drogas se convierte en el descriptor que más se relaciona con otros distintos, seguido de psicología y psicofarmacología. Se observa que el descriptor efecto de las drogas cobra más relevancia en este segundo periodo, ya que crece en co-presencias con otros descriptores distintos y en intensidad de sus relaciones. Siguen teniendo peso en esta red el tratamiento de la drogadicción y alcoholismo. En esta segunda red y en el período que abarca desaparece el descriptor síndrome de inmunodeficiencia adquirida y aparecen nuevos términos como salud laboral, neurociencias, genética clínica, emoción, motivación, neuroquímica y medida de la personalidad. 


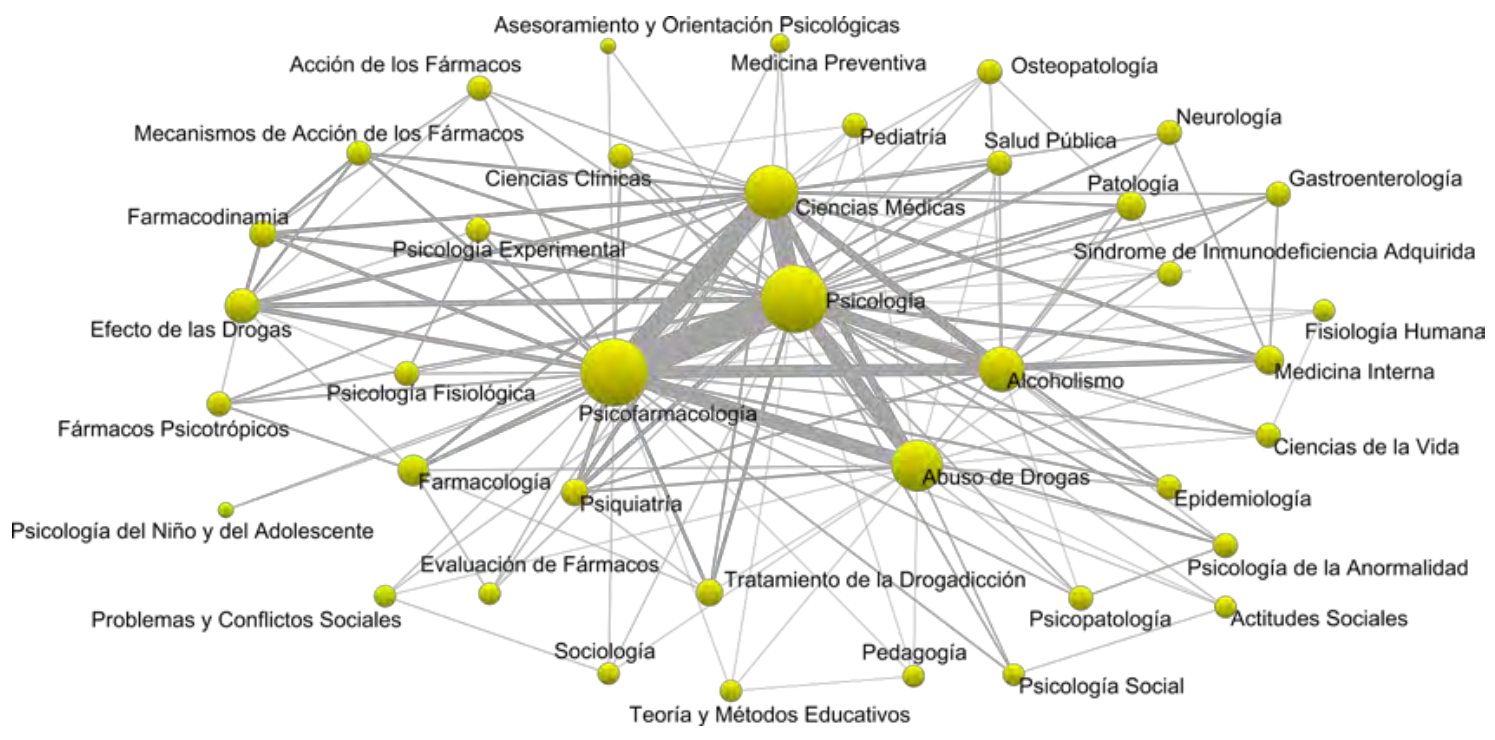

Figura 7. Red de co-ocurrencia de descriptores con tres o más co-presencias en las tesis doctorales sobre drogodependencias (1978-1997).

Nota. El diámetro de las esferas es proporcional a la cantidad de relaciones directas con otros descriptores y el grosor de las líneas representa el número de veces que dos descriptores coinciden en una misma tesis.

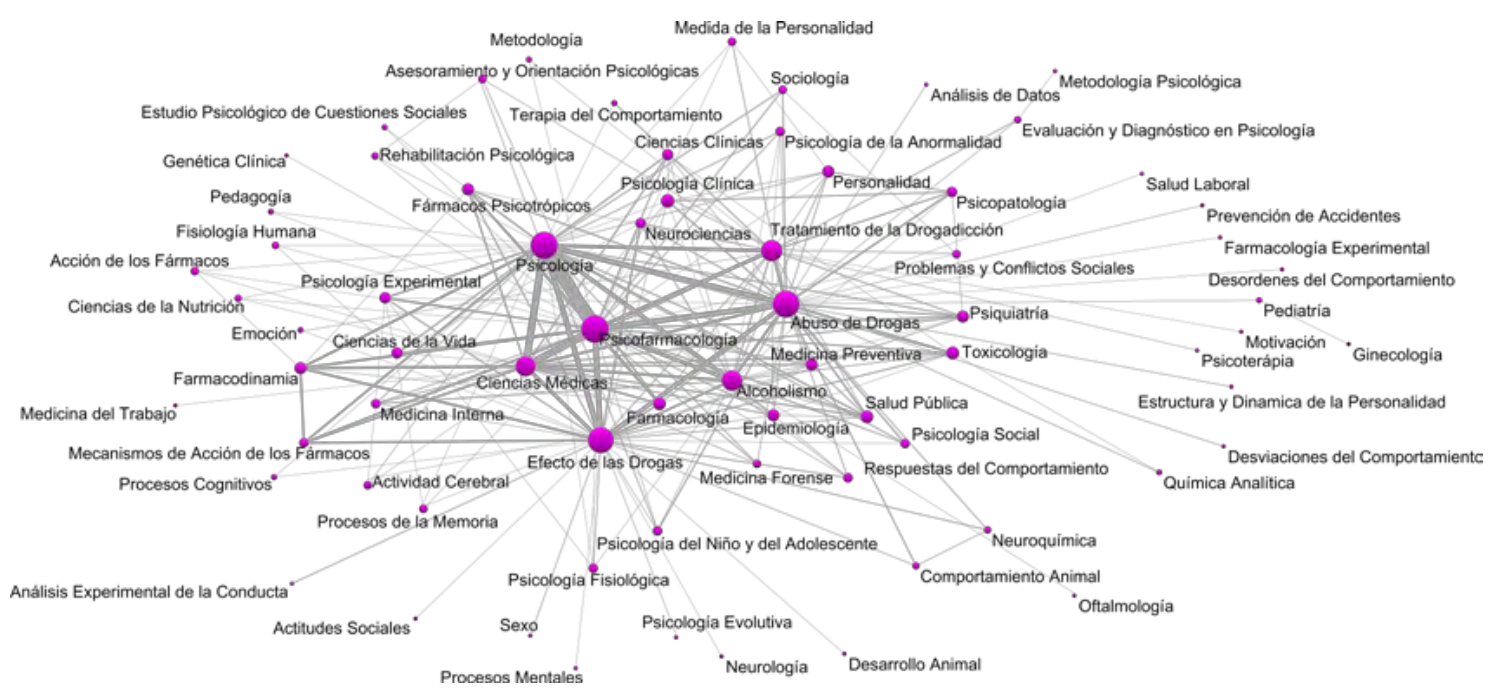

Figura 8. Red de co-ocurrencia de descriptores con tres o más co-presencias en las tesis doctorales sobre drogodependencias (1998-2017).

Nota. El diámetro de las esferas es proporcional a la cantidad de relaciones directas con otros descriptores y el grosor de las líneas representa el número de veces que dos descriptores coinciden en una misma tesis.

\section{Discusión}

La limitación más sustancial de este trabajo se debe a las carencias de la base de datos TESEO que, aun siendo la más completa en nivel de codificación de las tesis, se debe tener en cuenta que los datos de la ficha para el registro de las tesis son cumplimentados por los doctorandos o en la secretaría de la facultad o del departamento (Delgado et al., 2006). Por ello, los registros contienen, en ocasiones, errores y carecen de información relevante como, por ejemplo, que en algunos registros no estén codificados los tribunales o el director. A ello se suma las deficiencias en tanto la exhaustividad, calidad o texto completo, entre otras (Fuentes y Arguimbau, 2010). Otra de las limitaciones del estudio se refiere al hecho de que, aunque se han utilizado todos los descriptores UNESCO sobre el abuso de drogas, hay que tener en cuenta que puede haber sesgos, ya que es posible que existan tesis que traten sobre adicciones y no se hayan indizado por estos descriptores, o bien, 
que hayan tesis incluidas que correspondan a otro campo de estudio. Todos estos aspectos de deficiencia de codificación tienen como consecuencia la dificultad de recuperación o perdida de información (Curiel et al., 2018) valiosa y ligada a las adicciones y oscurece o emborrona las posibilidades de un mejor conocimiento del campo. Sin embargo, a pesar de todas estas limitaciones, la base de datos TESEO es una fuente eficaz para analizar la producción de tesis doctorales defendidas en las universidades españolas, ya que es la que más registros incluye (Machan y Sendra, 2018; Ramos-Pardo y Sánchez-Antolín, 2017).

Desde el inicio del nuevo siglo, los estudios sobre los trabajos de tesis doctorales han cobrado auge debido a que las tesis son un buen indicador para analizar la evolución de una ciencia, las líneas y tendencias de investigación o las personas más productivas, en tanto que dirigen tesis, pues se trata de un trabajo original y sujeto a un riguroso examen (Arguimbau-Vivó y Fuentes-Pujol, 2009; Curiel-Marín y Fernández-Cano, 2015; Delgado et al., 2006; Fuentes y Arguimbau, 2010; Fuentes-Pujol y González, 2002; Miguel, 2000; Repiso, Torres-Salinas y Delgado, 2011b). Asimismo, el estudio de las mismas, en una disciplina concreta, proporciona una visión de su estructura y de las personas más influyentes del campo analizado, ya que los alumnos suelen buscar como directores a los académicos de más prestigio o poder en el campo, pretendiendo con ello que el prestigio de este académico quede vinculado con su trabajo, ya que "la mera cantidad de tesis dirigidas basta para distinguir, en las diferentes disciplinas, a los grandes patrocinantes" (Bourdieu, 2008).

La producción de las tesis en el campo de las adicciones durante el periodo estudiado se asemeja a la mayoría de áreas de la ciencia donde la evolución de tesis defendidas experimenta una reducción en el curso académico 2004/2005, rompiendo así la tendencia de crecimiento sostenido mantenida hasta ese momento (Fuentes y Arguimbau, 2010). Esta reducción en casi todas las áreas se puede explicar por la deficiencia en TESEO en relación con la asignación de palabras claves, pues durante el periodo 2001-2005 existen numerosos registros que no las tienen asignadas (Sánchez et al., 2017), dando lugar a una pérdida de registros si se hacen las búsquedas en este campo, como es el caso. Sin embargo, en otras áreas, como la Sociología el crecimiento de defensa de tesis doctorales es constante (Castelló-Cogollos, 2016). El presente estudio sí que se asemeja a la Sociología en el crecimiento exponencial de los últimos quinquenios, fenómeno que no ocurre en otros campos como, por ejemplo, el de otorrinolaringología (Diego y Prim, 2008), el de la psicología (Agudelo et al., 2003; Osca-Lluch, Haba, Fonseca, Civera y Tortosa, 2013) o en el campo de la radio (Repiso et al., 2011b). Este crecimiento se podría explicar por la manifiesta preocupación que ha adquirido la problemática de las drogodependencias en los últimos años.
En relación con los autores de las tesis, se ha constatado que el $11,7 \%$ del total ha continuado con la carrera académica puesto que posteriormente han participado como directores o tribunales, 1,6 puntos por debajo del área de Sociología que se sitúa en 13,4\% (Castelló-Cogollos, 2016). Una posible explicación está en el perfil de los doctorados de esta área, ya que la mayoría de médicos y psicólogos, una vez egresados, se dedican a la asistencia clínica y no a la docencia universitaria o a la investigación.

La universidad que más tesis han producido sobre drogodependencias es la Universitat de Valencia probablemente porque tiene en sus planes de estudio diversos másteres dedicados a este campo, además de contar con grupos de investigación específicos como la Unidad de Investigación de Psicobiología de las Drogodependencias adscrita al Departamento de Psicología o el grupo de Neurofarmacología de la adicción (NEUROPHARAD) del Departamento de Farmacia. La Universitat de Valencia, la Universidad de Valladolid y la Universidad de Santiago de Compostela se sitúan entre las Universidades más productivas desbancando a las Universidades que constituyen la pentarquía en la producción de tesis doctorales en España y que la forman la Universidad Complutense de Madrid, la Universidad Autónoma de Madrid, la Universidad de Barcelona, la Universidad Autónoma de Barcelona y la Universidad de Granada (Sánchez et al., 2017).

En lo referente a los directores, es significativa la notable dispersión de personas que han dirigido tesis, ya que el $75,9 \%$ de los directores únicamente han dirigido una tesis, un valor que se sitúa casi 14 puntos porcentuales por debajo del observado en las tesis doctorales sobre estudios de las mujeres a nivel nacional en el período 1976-2002 (Torres y Torres, 2005),. Sin embargo, en áreas como la Biblioteconomía y Documentación únicamente seis directores acumulan casi el $50 \%$ de las tesis defendidas en España en el período 1992-2006 (Olmeda-Gómez, Perianes-Rodríguez, Ovalle-Perandones y de Moya-Anegón, 2009). Esta dispersión puede estar relacionada con la diversidad temática observada en el área de las adicciones, en tanto el consumo de sustancias afecta a multitud de aspectos sanitarios, familiares y sociales y, por consiguiente, pueden ser analizadas desde diversas vertientes metodológicas y teóricas.

$\mathrm{Si}$ se tienen en cuenta los directores de las tesis y los miembros de los tribunales se pueden extraer a los académicos más relevantes del campo de las adicciones. Por una parte, a nivel de directores, es clara la diferencia entre Alfonso Velasco Martín y el resto de directores. Velasco ha dedicado toda su carrera al estudio de los fármacos y los venenos en la Universidad de Valladolid, convirtiéndose en una autoridad en su campo. La mujer que más tesis doctorales ha dirigido es Ana María Bermejo Barrera que ha desarrollado su carrera en el campo de la toxicología en la Universidad de Santiago de Compostela. El hecho de que sólo ella se encuentre entra los diez principales directores puede ser debido a la 
diferencia de género ya que, como en diversas investigaciones se ha demostrado, las mujeres se encuentran con obstáculos relacionados con su naturaleza a la hora de ejercer sus profesiones (Alonso-Arroyo, Bolaños-Pizarro, González-Alcaide, Villamón y Aleixandre-Benavent, 2010; Vicente, 2003; García de Cortázar y García de León, 1997; García, 1990; Gonzalez-Alcaide, Agullo-Calatayud, Valderrama-Zurián y Aleixandre-Benavent, 2009; Lluch, 2012).

Por lo que respecta a las participaciones en los tribunales, existe una sustancial diferencia a la hora de participar en ellos, ya que no tiene el mismo valor participar como presidente que como vocal o secretario. Ser presidente tiene más responsabilidad o prestigio y, por esto, se suele nombrar a un académico de renombre en el campo. Si bien es cierto que este modelo de elección del presidente es el más extendido, se ha de tener en cuenta que en ocasiones se elige en función de la edad de las personas que forman el tribunal. Con todo, si se tiene en cuenta estas diferencias de peso en la participación, las posiciones cambiarían, ya que, aunque Emilio Ambrosio es el que más ha asistido a tribunales, la mayoría son como vocal y, sin embargo, Francesc Jané Carrenca casi la totalidad de sus asistencias son como presidente. En este caso, y bajo estas variables, se ha de tener en cuenta que Francesc Jané tiene una larga carrera en la medicina y es catedrático emérito de farmacología de la Universitat Autònoma de Barcelona.

Por lo tanto, el estudio de los directores y miembros de los tribunales, muestra que Alfonso Velasco o Francesc Jané tienen su carrera académica consolidada o terminada y, por lo tanto, con mayor prestigio y capacidad de acción, hecho que viene a confirmar la teoría de Bourdieu (2008) sobre que el acopio de capital y prestigio está directamente relacionado con los años en el mundo académico y con la edad. Resalta también el nombre de Miguel Sánchez Turet, que fue catedrático del Departamento de Psiquiatría y Psicología Clínica y fundador del máster en drogodependencias de la Universitat de Barcelona, y el de Elisardo Becoña Iglesias, catedrático de psicología clínica en la Universidad de Santiago de Compostela.

En cuanto al nivel de invitaciones al mismo académico a participar en los tribunales, se ha observado que el máximo es de 11 y el máximo de reciprocidad es de cinco y tres peticiones, que es muy similar al del campo de Sociología donde el máximo grado de invitación a la misma persona es de 10 (Castelló-Cogollos, 2016). Este dato de grado de invitación viene a sumarse a la dispersión existente en la asistencia a tribunales para ratificar que en el área de las drogodependencias no existe un nivel alto de endogamia y es similar al de la Sociología. Por tanto, se puede considerar que es un campo de la ciencia abierto a otras disciplinas y de intercambio de teorías y metodologías. Aún siendo así, la existencia de vínculos puede sugerir la existencia de un sistema de obligaciones, pues "aquel que solicita a un colega la participación en el jurado de una tesis que él ha dirigido se compromete tácitamente a conceder la reciprocidad y entra, por lo tanto, en un circuito de intercambios continuos" (Bourdieu, 2008).

Acerca de las comparativas del sexo de los autores, es a partir del quinquenio 1998-2002 cuando la proporción de las mujeres que se doctoran es mayor que el de hombres. Un hecho, observado en otras áreas como, por ejemplo, en Ciencias Experimentales y de la Salud, áreas en las que en el quinquenio 2006-2010 el porcentaje osciló por curso entre el $51 \%$ y el $56 \%$ (Instituto Nacional de Estadística; INE, 2011) o en la Psicología donde en el período de 1978 a 2012, las mujeres realizaron el 56,52\% de las tesis (Osca-Lluch et al., 2013). También en el ámbito de la Sociología en el periodo 2010-2013 el porcentaje de mujeres asciende a 57,81\% (Castelló-Cogollos, 2016).

En el caso de las directoras y tribunales, los porcentajes cambian, siendo los hombres los que alcanzan unas cuotas más elevadas a lo largo de todo el periodo de estudio, situándose en 25,6\% de mujeres en tribunales y 31,8\% de mujeres directoras. Estos datos son similares a los encontrados en otras áreas como la Sociología, donde el porcentaje de mujeres que han formado parte de los tribunales ha sido del 26,8\% y el de directoras del 17,2\% (Castelló-Cogollos, 2016); la Educación con un 32,7\% de mujeres en tribunales y un $36,4 \%$ de directoras (Moreno-Fernández y Moreno-Crespo, 2016); y la Psicología, con un 26\% y un $30 \%$, respectivamente (Osca-Lluch et al., 2013).

Si bien se observa un incremento paulatino de la presencia de las mujeres como directoras de las tesis y en los tribunales, el porcentaje de ellas en el último quinquenio sigue siendo inferior en 20 puntos porcentuales al de los hombres. Estos datos corroboran que la participación de la mujer en la educación va progresivamente en aumento y su consolidación, como alumnas y tituladas, es una realidad (Barona, 2013; Bermúdez et al., 2011; Lluch, 2012). Sin embargo, a partir de esta etapa, las carreras académicas de las mujeres se frenan y, un número elevado de ellas abandonan la trayectoria académica (Castelló-Cogollos, 2016). Dicho de otra manera, "el rendimiento escolar global" de las mujeres es superior en todos los niveles educativos (Manassero y Vázquez, 2003), en cambio, esta ventaja de las mujeres no se ve reflejada en los puestos académicos (Escribano, 2010; Lluch, 2012; Vicente, 2003), y se ha observado que entre los autores más productivos del área de las drogodependencias no existe igualdad de género (González-Alcaide at al., 2007). Sobre las causas de estas situaciones se ha escrito mucho y se han acuñado distintos términos que darían explicación a esta situación, como, por ejemplo, "cuello de botella", "tubo perforado", "histéresis" o "discriminaciones ocultas" (Diaz, 2008; Gonzalez-Alcaide et al., 2009). Sin embargo, la metáfora más utilizada para clarificar este fenómeno es el de "techo de cristal", el cual hace referencia a las barreras invisibles que discriminan a las mujeres, difíciles de localizar, 
pues no se encuentran en ninguna ley ni código social claramente identificable (García de Cortázar y García de León, 1997).

La universidad no puede dejar de incorporar a ese grupo de mujeres altamente cualificadas que contribuirían a la excelencia en la docencia y en la investigación. Para que esta situación avance y la igualdad de la mujer no sea solamente formal y retórica (Lluch, 2012), algunos trabajos (Díaz y Dema, 2013; Lluch, 2012) plantean algunas soluciones como la necesidad de aplicar medidas correctoras (generales, de maternidad y de acción positiva). Un ejemplo sería la supervisión de los procesos de selección, la parada por el reloj biológico o el reclutamiento activo de mujeres (Díaz y Dema, 2013), además de implantar y realizar el seguimiento, de forma efectiva, de las leyes sobre género, proyectándolas de manera transversal en todos los aspectos públicos (Lluch, 2012).

Por otra parte, y como era de esperar, la colaboración entre la psicología con la psicofarmacología y con el abuso de drogas es la más significativa, ya que históricamente "la intervención de la psicología en drogodependencias ha tenido un desarrollo paralelo al de la especialización en este ámbito" (Colegio Oficial de Psicólogos, 1998). Por un lado, el campo de las adicciones se beneficiaba de la profesionalidad y versatilidad para la intervención que tenían los psicólogos y, por otro lado, el campo de la psicología tenía un campo emergente donde desarrollar sus técnicas (Martín González, 2000).

Sin embargo, cuando se centra la atención en la evolución de la investigación sobre drogodependencias, llama la atención el cambio drástico de focos de atención. En un primer momento se centran en la psicofarmacología, en poder crear fármacos para tratar el problema de las adicciones y sus efectos, pero a partir del 2000 estas tendencias cambian y cae por completo la investigación en este aspecto, subiendo paulatinamente el interés en investigar el efecto de las drogas en el cerebro y como afectan al comportamiento humano. Este cambio se puede explicar por la influencia de distintas entidades y estamentos como el NIDA (National Institute on Drug Abuse) que, de la mano de Nora Volkow favorecieron la creación de un modelo de "enfermedad cerebral en adicciones". Este nuevo modelo queda consolidado en 2007 cuando en NIDA define la adicción como "una enfermedad crónica del cerebro (...) se considera una enfermedad del cerebro porque las drogas cambian el cerebro: modifican su estructura y como funciona" (Secades-Villa, García-Rodríguez, Fernández-Hermida y Carballo, 2007) y ha supuesto el incremento de la investigación en neurociencias, neuroquímica y genética clínica.

En definitiva, los resultados corroboran que el análisis de redes sociales y bibliométrico de las tesis en un campo específico de la ciencia, en relación a los directores y miembros de los tribunales, proporciona un instrumento que facilita la visión de su estructura, así como de las personas más influyentes y con mayor capacidad de acción en él (Bourdieu, 2008).

\section{Conclusiones}

De la realización de este estudio se desprende que el análisis de las tesis de una disciplina proporciona una visión de su estructura y de las personas más influyentes de la misma. Asimismo, se ha observado el uso de la base de datos TESEO tiene algunas limitaciones y las más significativas se relacionan con carencias en la codificación de los datos.

En cuanto a la realización de tesis se advierte que el incremento de la preocupación social por las consecuencias de las adicciones y el aumento de la creación de grupos y unidades de investigación que analicen esta problemática tienen su efecto en el incremento de la producción científica en el campo de las adicciones.

Los resultados indican que las posiciones con más poder de acción y prestigio académico las ocupan profesores como Alfonso Velasco o Frances Jané, como era de esperar, pues en unos casos han finalizado su carrera docente o ésta está bien consolidada. Pero también han sido o son académicos relevantes en el campo de las drogodependencias Emilio Ambrosio Flores, Miguel Sánchez Turet o Elisardo Becoña Iglesias.

En los cálculos de las instituciones se aprecia que las universidades de referencia en este ámbito son la Universitat de València, la Universitat Autònoma de Barcelona y la Universidad de Valladolid.

En lo relativo al estudio de género, el ascenso de las mujeres a los puestos de gran responsabilidad académica o de poder es lento, pues los niveles de participación tanto como directoras como miembros de tribunales continúa siendo inferior al de los hombres. Por consiguiente, se puede decir que en el ámbito universitario también existe, en esta área, un "techo de cristal" que dificulta que las mujeres progresen en el ámbito laboral, obstruyendo el acceso a los puestos elevados de responsabilidad.

Por lo que respecta a las tendencias investigadoras en drogodependencias se ha observado un vuelco, ya que al principio se centro en la psicofarmacología y sus efectos y posteriormente se focaliza en los efectos de las drogas en el cerebro. De igual modo, el gran número de descriptores encontrados en el presente estudio es una muestra de la amplia diversidad de aspectos a los que afectan las drogodependencias.

\section{Agradecimientos}

Este trabajo se ha realizado con la ayuda del Plan Municipal de Drogodependencias de la Concejalía de Sanidad, Salud y Deportes del Ayuntamiento de Valencia, España. 


\section{Conflicto de intereses}

Los autores de este artículo declaramos que no existe conflicto de intereses.

\section{Bibliografía}

Agudelo, D., Bretón-López, J., Ortiz-Recio, G., Poveda-Vera, J., Teva, I., Valor-Segura, I. y Vico, C. (2003). Análisis de la productividad científica de la psicología española a través de las tesis doctorales. Psicothema, 15, 595-609.

Alonso-Arroyo, A., Bolaños-Pizarro, M., González-Alcaide, G., Villamón, M. y Aleixandre-Benavent, R. (2010). Análisis de género, productividad científica y colaboración de las profesoras universitarias de ciencias de la salud en la Comunidad Valenciana (2003-2007). Revista Española de Documentación Científica, 33, 624-642. doi:10.3989/ redc.2010.4.764.

ANECA (2017). Criterios de evaluación [noviembre-2017]. Recuperado de http://www.aneca.es/Programas-de-evaluacion/Evaluacion-de profesorado/ACADEMIA/Criterios-de-evaluacion-noviembre-2017.

Arguimbau-Vivó, L. y Fuentes-Pujol, M. E. (2009). Tesis doctorales en España: análisis de la bibliografía específica y su accesibilidad. Ibersid, 95-104. Recuperado de https://www.ibersid.eu/ojs/index.php/ibersid/article/ download/3729/3490.

Barona, J. L. (2013). Mujeres y ciencia, genealogía de una exclusión. Mètode Science Studies Journal - Annual Review, 76, 51-55. doi:10.7203/metode.76.2063.

Bermúdez, M. P., Guillén-Riquelme, A., Gómez-García, A., Quevedo-Blasco, R., Sierra, J. C. y Buela-Casal, G. (2011). Análisis del rendimiento en el doctorado en función del sexo. Educación XX1, 14, 17-33.

Bourdieu, P. (2008). Homo academicus. Madrid: Siglo XXI.

Bramness, J. G., Henriksen, B., Person, O. y Mann, K. (2014) A bibliometric analysis of European versus USA research in the field of addiction. Research on alcohol, narcotics, prescription drug abuse, tobacco and steroids 2001. European Addiction Research, 20, 16-22. doi:10.1159/000348260.

Buela, G. (2005). El sistema de habilitación nacional: criterios y proceso de evaluación. Análisis y Modificación de Conducta, 31, 313-345.

Caparrós-Ruiz, A. (2018). Time to the doctorate and research career: some evidence from Spain. Research in $\mathrm{Hi}$ gher Education. doi:10.1007/s11162-018-9506-2.

Castelló-Cogollos, L. (2016). El campo de la sociología en España (1976-2013). Análisis sociométrico y de redes de las tesis doctorales y sus tribunales (tesis doctoral). Universidad de Valencia, Valencia, España.

Castelló-Cogollos, L., Bueno, F. J. y Aleixandre, R. (2016). Tendencias de investigación en las tesis españolas bobre drogodependencias en la base de datos TESEO (19782016) [Internet]. Recuperado de: http://cendocboga-
ni.org/Archivos/TemasActualidad/Tendencias-investigación-tesis-españolas-drogodependencias-TESEO.pdf

Colegio Oficial de Psicólogos. (1998). Perfiles profesionales del psicólogo. Madrid: Colegio Oficial de Psicólogos.

Curiel-Marín, E. y Fernández-Cano, A. (2015). Análisis cienciométrico de tesis doctorales españolas en didáctica de las ciencias sociales ( 1976-2012 ). Revista Española de Documentación Científica, 38, 1-10. doi:10.3989/ redc.2015.4.1282.

Curiel-Marín, E., Passoni, L. I., Olmedo-Moreno, E. M. y Fernández-Cano, A. (2018). Los mapas auto-organizados para la evaluación de la investigación de tesis doctorales: el caso de la didáctica de las ciencias sociales en España. RELIEVE, 24, 1-17. doi: 10.7203/relieve.24.1.12345.

CRUE Universidades Españolas. (2017). Las universidades españolas en cifras. Recuperado de http://www.crue.org/ SitePages/La-Universidad-Española-en-Cifras.aspx.

Davis, A., Wladkowski, S. P. y Mirick, R. G. (2017) Lessons learned for successful dissertation completion from social work doctoral graduates. Journal of Teaching in Social Work, 37, 107-120. doi:10.1080/08841233.2017.1295124.

Delgado, E., Torres-Salinas, D., Jiménez-Contreras, E. y Ruiz-Pérez, R. (2006). Análisis bibliométrico y de redes sociales aplicado a las tesis bibliométricas defendidas en España (1976-2002): temas, escuelas científicas y redes académicas. Revista Española de Documentación Científica, 29, 493-524. doi:10.3989/redc.2006.v29.i4.306.

Diaz, C. (2008). ¿Por que es tan lento el progreso de las mujeres en la carrera científica? SEBBM, 158, 5-8. Recuperado de http://www.sebbm.com/pdf/158/d01158.pdf.

Díaz, C. y Dema, S. (2013). La escasez de mujeres en la academia. Un caso de histéresis social.100cias@uned,6, 149-156. Recuperado de http://e-spacio.uned.es/fez/ eserv/bibliuned:revista100cias-2013-6ne-2025/Mujeres_histeresis.pdf.

Diego, J. I. y Prim, M. P. (2008). Análisis de las tesis doctorales en otorrinolaringología presentadas en España en el período 1976-2005. Acta Otorrinolaringológica Española, 59, 292-297. doi:10.1016/S0001-6519(08)73313-2.

Escribano, P. (2010). Mujeres en, por y para la ciencia. Dossieres Feministes, 14, 151-174. Recuperado de https:// www.raco.cat/index.php/DossiersFeministes/article/ download/229295/311005.

European Monitoring Centre for Drugs and Drug Addiction. (2018). Publications. Lisboa: European Monitoring Centre for Drugs and Drug Addiction. Recuperado de http:/ /www.emcdda.europa.eu/publications_en.

Fuentes, E. y Arguimbau, L. (2010). Las tesis doctorales en España (1997-2008): análisis, estadísticas y repositorios cooperativos. Revista Española de Documentación Científica, 33, 63-89.

Fuentes-Pujol, E. y González, A. (2002). Tesis doctorales en España en información y documentación, 19762001. En M. A. Morán Suárez y M. C. Rodríguez López 
(Coord.). La documentación para la investigación: homenaje a José Antonio Martín Fuertes (pp. 229-241). León: Universidad de León.

García de Cortázar M. L. y García de León, M. A. (1997). Mujeres en minoría: una investigación sociológica sobre las catedráticas de universidad en España. Madrid: Centro de Investigaciones Sociológicas.

García, V. (1990). Reflexiones sobre la accion y la influencia docentes: maestros y profesores. Anales de la Real Academia de Ciencias Morales y Políticas, 42, 35-45.

González-Alcaide, G., Agulló-Calatayud, V., Valderrama-Zurián J. C. y Aleixandre-Benavent, R. (2009). Participacion de la mujer y redes de coautoria en las revistas españolas de sociología. Revista Española de Investigaciones Sociologicas, 126, 153-166.

Gonzalez-Alcaide, G., Arroyo-Alonso, A., Valderrama-Zurián, J. C. y Aleixandre-Benavent, R. (2009). Mujeres en la investigacion cardiológica española. Revista Española de Cardiologia, 62, 945-946. doi:10.1016/S03008932(09) 72082-2.

González-Alcaide, G., Bolaños-Pizarro, M., Navarro-Molina, C., De Granda Orive, J. I., Aleixandre-Benavent, R. y Valderrama-Zurián, J. C. (2008). Análisis de la producción científica española sobre abuso de sustancias en función de la colaboración entre disciplinas. Adicciones, 20, 337. doi:10.20882/adicciones.737.

González, G., Fernandes, L., Pinto, M. y Agulló, V. (2015). La investigación biomédica sobre abuso de sustancias a través de la base de datos Medline (2006-2010). Revista Española de Drogodependencias, 40, 29-45.

González-Alcaide, G., Valderrama-Zurián, J.C., Navarro-Molina, C., Alonso-Arroyo, A., Bolaños-Pizarro, M. y Aleixandre-Benavent, R. (2007). Análisis de género de la producción científica española sobre drogodependencias en biomedicina 1999-2004. Adicciones, 19, 45-50. doi:10.20882/adicciones.322.

Instituto Nacional de Estadística; INE. (2012). Estadística de enseñanza universitaria. Resultados nacionales, por comunidades autónomas y provincias. Recuperado de http:/ / www.ine.es/dyngs/INEbase/es/operacion.ht$\mathrm{m}$ ?c=Estadistica_C\&cid=125473617644\&menu=resultados\&idp=1254735573113.

Khalili, M., Rahimi-Movaghar, A., Shadloo, B., Mojtabai, R., Mann, K. y Amin-Esmaeili, M. (2018). Global scientific production on illicit drug addiction: A two-decade analysis. European Addiction Research, 24, 60-70. doi:10.1159/000487590.

Lluch, A. (2012). El camí cap a la igualtat real. La precència de la dona en l'ámbit acadèmic. Mètode, 75, 77-87. Recuperado de https://metode.cat/revistes-metode/ monografics/cami-cap-a-igualtat-real.html.

Machan, K. y Sendra, F. (2018). Doctoral theses in diagnostic imaging: a study of Spanish production between 1976 and 2011. Radiología. doi:10.1016/j.rxeng.2018.07.002.
MacLennan, H. L., Piña, A. y Gibbons, S. (2018). Content analysis of DBA and PhD dissertations in business. Journal of Education for Business. doi:10.1080/08832323.201 8.1438983 .

Manassero, M. y Vázquez, Á. (2003). Los estudios de género y la enseñanza de las ciencias. Revista de Educación, 97, 251-279.

Martín, E. (2000). Psicología y drogas. Aproximación histórica, situación actual y perspectivas de futuro. Papeles del Psicólogo, 77, 2-13.

Melero-Fuentes, D. (2016). Análisis de la producción científica en sustancias adictivas a través de web of science durante el periodo 2008-2012 (tesis doctoral). Universidad Católica de Valencia San Vicente Mártir, Valencia, España.

Miguel, A. (2000). Aportaciones al estudio de la literatura gris universitaria: la evolución de la tesis doctoral en España. En I Congreso Universitario de Ciencias de la Documentación: Teoría, historia y metodología de las Ciencias de la Documentación. (pp. 431-437). Madrid: Universidad Complutense de Madrid.

Moreno-Fernández, O. y Moreno-Crespo, P. (2016). Análisis bibliométrico de las tesis doctorales españolas indexadas con el descriptor "sector de la educación" (1976/2014). Revista Española de Documentación Científica, 39, e146. doi.org/10.3989/redc.2016.3.1331.

Olmeda-Gómez, C., Perianes-Rodríguez, A., Ovalle-Perandones, M. A. y de Moya-Anegón, F. (2009). Colegios visibles: estructuras de coparticipación en tribunales de tesis doctorales de biblioteconomía y documentación en España. El Profesional de la Información, 18, 41-49. doi: 10.3145/epi.2009.ene.06

Osca-Lluch, J. (2012). Productividad y colaboración científica desde una perspectiva de género en la Revista Española de Drogodependencias. Revista Española de Drogodependencias, 37, 9-21.

Osca-Lluch, J. (2016). Producción científica en consumo de cannabis y tabaco a través de la Web of Science. Informe ÉVICT, 2, 26-40. Recuperado de http:/ / evictproject. org/wp-content/uploads/2015/06/Produccion-cientifica-Web-of-Science.pdf.

Osca-Lluch, J., Haba, J., Fonseca, S., Civera, C. y Tortosa, F. M. (2013). Tesis doctorales españolas sobre análisis bibliométrico en psicología. Aula Abierta, 41, 99-110.

Piña, A. A., MacLennan, H. L., Moran, K. A. y Hafford, P. F. (2016). The DBA vs. PhD in U.S. business and management programs: Different by degrees? Journal of Excellence in Business Education, 4, 6-19.

Ramos-Pardo, F. J. y Sánchez-Antolín, P. (2017). Production of educational theory doctoral theses in Spain (2001-2015). Scientometrics, 112, 1615-1630. doi:10.1007/ s11192-017-2435-6.

Real Decreto 99/2011, de 28 de enero, por el que se regulan las enseñanzas oficiales de doctorado. Boletín Oficial del Estado núm 35, del 10 de febrero de 2011, 
pp. 13909-13926. Recuperado de https://www.boe.es/ buscar/act.php?id=BOE-A-2011-2541.

Repiso, R., Torres, D. y Delgado, E. (2011a). Análisis bibliométrico y de redes sociales en tesis doctorales españolas sobre televisión (1976/2007). (Spanish). Comunicar, 19, 151-159. doi:10.3916/C37-2011-03-07.

Repiso, R., Torres-Salinas, D. y Delgado, E. (2011b). Análisis de la investigación sobre radio en España: una aproximación a través del análisis bibliométrico y de redes sociales de las tesis doctorales defendidas en España entre 1976-2008. Estudios sobre el Mensaje Periodístico, 2, 417-429.

Round University Ranking. (2018). RUR World University Rankings. Recuperado de http://roundranking.com/ ranking/world-university-rankings.html\#world.

Sánchez, R., Blázquez, M., Montesi, M. y Botezan, I. (2017). La producción de tesis doctorales en España (19952014): evolución, disciplinas, principales actores y comparación con la producción científica en WoS y Scopus. Revista Española de Documentación Científica, 40, e188. doi: 10.3989/redc.2017.4.1409.

Secades-Villa, R., García-Rodríguez, O., Fernández-Hermida, J. R. y Carballo, J. L. (2007). Fundamentos psicológicos del tratamiento de las drogodependencias. Papeles del Psicólogo, 28, 29-40.

Times Higher Educación. (2018). The World University Rankings. Recuperado de https://www.timeshighereducation.com/world-university-rankings.

Torres, I., y Torres, D. (2005). Tesis doctorales sobre estudios de las mujeres en España (1976-2002). A propósito de un indicador definitivo en investigación. Revista Espanola de Documentación Científica, 28, 479-499.

U-Multirank. (2018). U-Multirank. Universities compared. Your way. Recuperado de https://www.umultirank.org.

Vicente, G. (2003). Mujeres en el mundo académico español. Thémata. Revista de Filosofia, 31, 173-185.

Vidal-Infer, A. M. (2010). Análisis de los artículos originales publicados en revistas específicas sobre drogodependencias incluidas en el Journal Citation Reports (2002-2006) (tesis doctoral). Universitat de València, Valencia, España. 